\title{
The respiratory effects of occupational polypropylene flock exposure
}

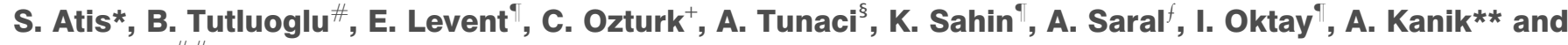 \\ B. Nemery $\# \#$
}

ABSTRACT: The present study evaluated the possible effects of exposure to polypropylene flock on respiratory health and serum cytokines in a cross-sectional study of workers from a plant in Turkey.

A total of $\mathbf{5 0}$ polypropylene flocking workers were compared to a control group of 45 subjects. All subjects filled out a respiratory questionnaire and underwent a physical examination, a chest radiograph and pulmonary function testing, including single breath carbon monoxide diffusing capacity ( $D$ L,CO). Serum interleukin-8 (IL-8) and tumour necrosis factor-alpha (TNF- $\alpha$ ) were measured. Additionally, high resolution computed tomography (HRCT) of the chest was performed in 10 exposed workers with low DL,Co.

Work-related respiratory symptoms were reported in $26 \%$ of the exposed subjects and in $13.3 \%$ of the controls. Logistic regression analysis showed that the risk of respiratory symptoms increased 3.6 fold in polypropylene flocking workers when compared to controls. Parameters of the study group, including per cent predicted: forced vital capacity, forced expiratory volume in one second, forced mid-expiratory flow $25-75 \%$ and $D L, C O$, were significantly lower than in controls. Multivariate analyses showed that being a polypropylene flocking worker was a predictive factor for impairment of pulmonary function. Serum IL-8 and TNF- $\alpha$ levels were increased in the study group compared with the controls. HRCT revealed peribronchial thickening and diffuse ground glass attenuation in some subjects.

The present study suggests the presence of subtle or the beginning of interstitial lung disease in these polypropylene flocking workers.

KEYWORDS: Cytokine, interstitial lung disease, lung function, occupational lung disease, polypropylene flock, respirable dust

$\mathbf{R}$ ecently, there has been concern about the potential health hazard from occupational exposure to synthetic polymers inhaled as microfibres or "flock". Since 1999, several cases of a particular occupational interstitial lung disease (ILD), termed "flock worker's lung", have been reported among workers exposed to nylon flock in the USA and Canada [1-3]. In Europe, BARROSO et al. [4] reported a case of ILD in a woman who had had a longstanding occupational exposure to polyethylene flock.

Flock is composed of synthetic or natural short fibres $(0.2-5 \mathrm{~mm})$, which have been generally cut from tow (a loose rope of thin continuous strands), and is applied to an adhesive-coated substrate. Synthetic materials that produce flock include nylon, rayon, polyester, and polyolefins $[2,3,5]$. Polyolefin fibres are made from polymers of propylene, ethylene or others olefins. About $95 \%$ of polyolefin fibres are composed of polypropylene. Polypropylene flock is used for the manufacture of plastic items including tows, fabrics, upholstery, carpets, fishing nets, bags and many other products [5].

Although, the exact aetiological agent for flock worker's lung is unknown, studies have suggested that aerosolised flock may contain respirable-sized particles that may lead to inflammatory reactions in the lung [6-9].

Various cytokines secreted from macrophages play a key role in the pathogenesis of ILDs [10, 11]. Increased levels of Interleukin-8 (IL-8) and tumour necrosis factor-alpha (TNF- $\alpha$ ) have been described in various inflammatory disorders characterised by neutrophilic infiltration, including interstitial pulmonary disorders [12, 13], but there are no published studies of flock worker's lung in which these cytokines have been measured.
AFFILIATIONS

*Dept of Chest Diseases,

${ }^{+}$Dept of Microbiology,

"Dept of Biostatistics, School of

Medicine, Mersin University, Mersin,

\#Dept of Chest Diseases,

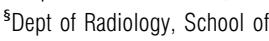

Medicine Cerrahpaşa, Istanbul

University, Istanbul,

'Tekirdag Chest Diseases Hospital, Tekirdag,

${ }^{f}$ Dept of Environment Engineering, Faculty of Civil Engineering, Yildiz

Technical University Istanbul,

Istanbul, Turkey.

\#\#K.U. Leuven Laboratorium voor

Pneumologie (Longtoxicologie),

Leuven, Belgium.

CORRESPONDENCE

S. Atis

Dept of Chest Disease

School of Medicine

Mersin University

33079

Mersin

Turkey

Fax: 903243374305

E-mail: satis@mersin.edu.tr

Received:

December 142003

Accepted after revision:

September 222004 
The published case series of flock worker's lung prompted us to investigate respiratory health in workers from the polypropylene flock processing industry and the present authors also attempted to verify the possible contribution of the proinflammatory mediators, IL- 8 and TNF- $\alpha$, in the pathogenesis of flock worker's lung.

\section{Plant description}

The production department of this plant consists of two distinct enclosed rooms, which are the extrusion and flocking rooms, each $\sim 1000-1500 \mathrm{~m}^{3}$. In the extrusion room, there were three extrusion machines in operation, which were continuously processing the polypropylene raw material in granular form, producing plastic tows. In the flocking room, there were 12 guillotine cutting machines in operation (fig. 1a). The polypropylene tows were cut by these machines which generated small size fibres (flock). The composition of the flock produced at this plant is $90 \%$ of polypropylene, $5 \%$ of polyethylene, $3 \%$ of antisplit, $1.5 \%$ of ultraviolet stabiliser, $0.25-0.50 \%$ of granule dye. This flock is used to produce new plastic fabrics for manufacturing bags and sacks. Flock is produced continuously in this plant, with employees working three 8-h shifts (fig. 1b). At the time of the present study, 58 workers were employed in the production department. The ventilating and air conditioning systems were inadequate
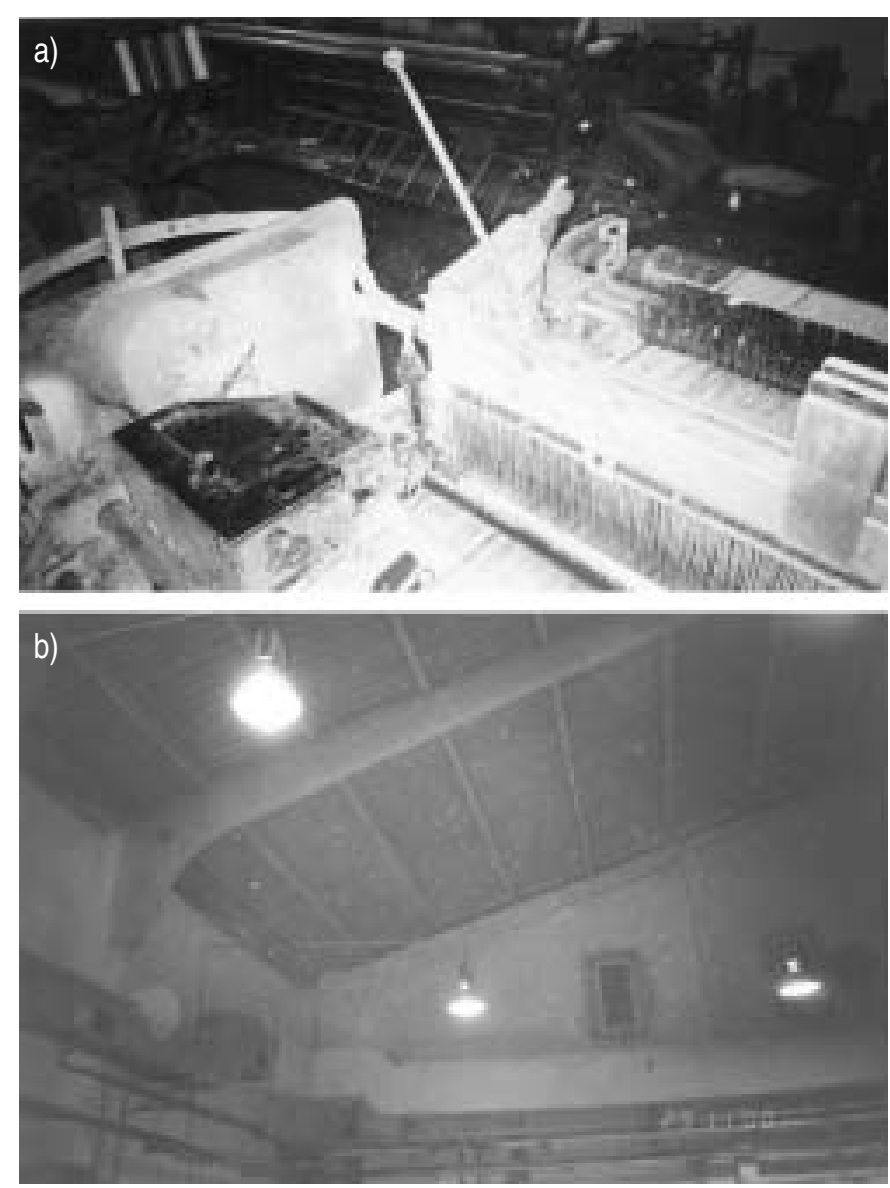

FIGURE 1. Polypropylene fibre fragments a) from a guillotine cutting machine and $b$ ) in the air of the flocking department of the plant. and no respiratory protective measures were taken in the department. The departments of control subjects were in a separate building, away from the production department.

\section{METHODS}

The present cross-sectional study was performed in September through to November 2001. Of the 58 workers, who worked in direct exposure to polypropylene flock in a polypropylene flock processing plant in Tekirdag, Turkey, 50 subjects accepted to participate. These workers were enrolled as the study group. They had been working for $\geqslant 3 \mathrm{yrs}$ in the same part of the factory. A total of 45 workers among the 60 who were not exposed to polypropylene flock but worked in other parts of the factory (security persons, drivers, workers in the package units, etc.) were randomly (with MINITAB's random sample command) included as the control group.

The current authors developed a detailed questionnaire by modifying a previous questionnaire from the literature $[6,14]$. This questionnaire included data on demographic factors, tobacco use, medication use, and respiratory symptoms (dyspnoea, cough, phlegm, wheezing or chest tightness). Questions on work-related respiratory symptoms, determined as "occurring during work more frequently than normal and improving when away from work" were also included. Additionally, participants were asked how many days they worked per week, how many hours they worked per workday, and how many years they had worked in the polypropylene flocking department. A physical examination, including chest auscultation, was performed on all workers.

\section{Pulmonary function and $\mathrm{CO}$ diffusing tests}

The pulmonary function tests, including the $D \mathrm{~L}, \mathrm{CO}$ measurements, were performed in the plant using computer-assisted spirometry (Vmax22D; Sensor Medics, CA, USA) in accordance with the European Respiratory Society's (ERS) recommendations. Measurements of forced vital capacity (FVC), forced expiratory volume in one second (FEV1), FEV1/FVC ratio, and maximal expiratory flow at $25-75 \%$ of vital capacity (FEF25-75\%) were obtained and expressed as per cent predicted (\% pred) using normal values for adults of the European Community for Steel and Coal [15]. Carbon monoxide (CO) diffusing tests were performed by the single-breath method, also in accordance with ERS recommendations, to obtain a diffusing capacity of $\mathrm{CO}(\mathrm{DL}, \mathrm{CO} \%)$ [16].

\section{Radiological investigation}

Standard posteroanterior chest radiographs of all workers were taken in the local hospital and evaluated by two chest physicians and two radiologists independently. Additionally, 10 polypropylene flocking workers, who had low $D \mathrm{~L}, \mathrm{CO} \%$, underwent both inspiratory and expiratory high-resolution computed tomography (HRCT). HRCT was performed with a spiral CT machine (Siemens Somatom Plus S; Siemens, Erlangen, Germany) using $1 \mathrm{~mm}$ collimation at $10 \mathrm{~mm}$ intervals and evaluated by two radiologists independently.

\section{Cytokine measurement}

PeliKine Compact human IL- 8 and human TNF- $\alpha$ ELISA kits (CLB, Amsterdam, the Netherlands) were used for assaying IL8 and TNF- $\alpha$ levels in serum. Measurements were made 
according to the kit procedure [17]. All values were expressed as $\mathrm{pg} \cdot \mathrm{mL}^{-1}$. The check of the ELISA kit is a standard curve of 10-240 $\mathrm{pg} \cdot \mathrm{mL}^{-1}$ for IL-8 and $0-1000 \mathrm{pg} \cdot \mathrm{mL}^{-1}$ for TNF- $\alpha$.

\section{Microscopic examination of polypropylene flock}

The diameter and length of 30 fibre samples of a polypropylene flock were measured using polarised light microscopy (Euromex binocular polarised microscopy, the Netherlands). Additionally, flock was examined by scanning electron microscopy. Samples were fixed with $2.5 \%$ phosphate-buffered glutaraldehyde at $4{ }^{\circ} \mathrm{C}$ for 9 days. After fixation, samples were washed with phosphate buffer and dehydrated with increasing concentrations of acetone (25, 50, 75 and 100\%). Dehydration was completed with a Jumbo Critical Point Dryer (CA, USA). Samples were coated with 200 Au-thick gold using gold coating apparatus and examined in a Jeol Sem EM-EX 1200 (Tokyo, Japan) at $80 \mathrm{kV}$.

\section{Dust measurements at the working environment}

Total (inhalable) and respirable dust measurements were carried out in the production department (in February 2004) according to the standard procedure stated in Occupational Safety and Health A (OSHA) Technical Manual TED 1-0,15A. Stationary sampling, using a SKC Brand 224-44XR model Universal Sampling Pump, was done (from 08:00 h to 17:00 h at consecutive hours) in the vicinity of the cutting machines, i.e. the area of highest visible dust pollution, even though the operators were not necessarily in these locations all the time. All measurements were carried out on a single day without regarding the time of the day since there is a continuous $(24 \mathrm{~h})$ production scheme in the plant. This brings out steady air conditions throughout all hours in terms of air pollutants. So, taking one air sample for a particular parameter at any time can be considered representative for the whole working day for that parameter. Therefore, one sampling was performed for each inhalable and respirable dust at two distinct locations in the plant. The inhalable dust sampling was performed using the Institute of Occupational Medicine (IOM) sampling cassette, and respirable dust sampling was performed using a cyclonic collector (SKC Conductive Plastic Cyclone 225-69 for respirable dust; Houston, TX, USA) having 5 micron cutpoint particle diameter at $1.9 \mathrm{~L} \cdot \mathrm{min}^{-1}$ constant sampling flow rate. All gravimetric measurements were performed using a digital balance having $0.1 \mathrm{mg}$ resolution.

\section{Statistical analyses}

Data are expressed as mean $\pm \mathrm{SD}$. In the comparison of pulmonary function test parameters between the study and control groups, an independent t-test is used. Additionally, multivariate analyses of variance (MANOVA) were performed to analyse the predictive factors for impairment of lung function parameters. In this model, pulmonary function test parameters (FEV1\%, FVC\%, FEV1/FVC, FEF25-75\%, and $\mathrm{DL}, \mathrm{CO} \%$ ) were used as dependent variables, while age, sex, tobacco use, working years, and the group of subjects served as covariates. Comparisons of serologic variables between the study and control groups were performed using the Mann Whitney U-test, because assumptions for the parametric test were invalid, and results were given as median with $25-75 \%$ interquartile ranges. Additionally, a repeatability test was performed for serologic measurements. Correlations between the serologic and pulmonary functional variables were determined by Spearman's rank correlation coefficient. Chi squared tests were used to compare the differences in the characteristics of two groups. Comparisons of each respiratory symptom between the study and control groups were performed using the $\mathrm{z}$ test for two proportions. Additionally, logistic regression analysis (with forward logistic regression method) was performed to determine the significant risk factors which have a role for development of any respiratory symptoms. A new variable was created which included at least one of the respiratory symptoms (dyspnoea, cough, phlegm, wheezing, or chest tightness). This new variable served as the dependent variable and age, sex, tobacco use, working years, and the group of subjects as covariates in logistic regression analyses. Statistical significance was accepted at the $p<0.05$ level.

\section{RESULTS}

There were 39 male and 11 female workers in the study group and 32 male and 13 female workers in the control group. Demographic characteristics of workers are presented in table 1 . There were no differences in age $(p=0.28)$, sex $(p=0.44)$ or smoking status $(p=0.83)$ between study and control subjects.

All participants reported working $8 \mathrm{~h}$ per day and 6 days per week in their department. The prevalence of respiratory symptoms in the study and control groups is shown in table 2.

\begin{tabular}{|c|c|c|}
\hline & Study group \# & Control group \\
\hline Age & $32.2 \pm 8.6$ & $33.9 \pm 6.1$ \\
\hline \multicolumn{3}{|l|}{ Sex } \\
\hline Female & 11 & 13 \\
\hline Male & 39 & 32 \\
\hline \multicolumn{3}{|l|}{ Smoking } \\
\hline Current smoker & $22(44)$ & 19 (42.3) \\
\hline Ex-smoker & $17(34)$ & $16(35.5)$ \\
\hline Nonsmoker & $11(22)$ & 10 (22.2) \\
\hline Working yrs & $9.6 \pm 4.9$ & $8.8 \pm 4.1$ \\
\hline
\end{tabular}

TABLE 2 Prevalence of respiratory symptoms in workers $\begin{array}{cc}\text { Study } & \text { Control } \\ \text { group }^{\#} & \text { group }\end{array}$

\begin{tabular}{lclc} 
Respiratory symptoms (ever) & & & \\
Dyspnoea & $12(34)$ & $5(11.1)$ & 0.102 \\
Cough & $8(16)$ & $3(6.6)$ & 0.156 \\
Wheeze & $2(4)$ & $1(2.2)$ & 0.621 \\
Chest pain & $1(2)$ & $0(0)$ & NA \\
Work-related respiratory symptoms & $13(26)$ & $6(13.3)$ & 0.123 \\
\hline
\end{tabular}

Data presented as $n$ (\%). NA: not applicable. ${ }^{\#}: n=50 ; ~ ': n=45$. 
Work-related respiratory symptoms were reported in $13(26 \%)$ of the study subjects and $6(13.3 \%)$ of the control subjects. This difference was not statistically significant $(p=0.123)$.

However, logistic regression analysis showed that the risk of reporting at least one respiratory symptom increased 3.6-fold in polypropylene flocking workers when compared to controls (odds ratio: 3.6; 95\% confidence interval (CI): 1.07-12.02). Age, sex, tobacco use, and working yrs were not found to be significantly associated with respiratory symptoms.

Physical examinations of the study and control groups were evaluated as normal.

The pulmonary function test variables are presented in table 3. The values for $\mathrm{FVC} \%, \mathrm{FEV} 1 \%, \mathrm{FEF} 25-75 \%$ and $\mathrm{DL}, \mathrm{CO} \%$ of workers exposed to polypropylene flock were significantly lower than in controls. However, FEV1/FVC did not differ between the two groups. Restrictive impairment (defined as FVC \% predicted less than the lower 95th percentile value of the control group with FEV1/FVC more than the higher 95th percentile value of the control group) was found in 10 of the 50 polypropylene flocking workers compared to 2 of $45(4.4 \%)$ controls $(\mathrm{p}=0.023)$. Low diffusing capacity (defined as $\mathrm{DL}, \mathrm{CO} \%$ predicted $<83.3 \%$, being the lower $95^{\text {th }}$ percentile value of control) was observed in 13 of 50 (26\%) polypropylene flocking workers compared to 2 of $45(4.4 \%)$ of the control group $(\mathrm{p}=0.004)$.

In the MANOVA, being a worker in the polypropylene flock industry $(p=0.001)$ and the duration of work in yrs $(p=0.03)$ were found to be predictive factors for impairment of lung function. Tobacco use was not found to be a predictive factor for the impairment of pulmonary function in this model.

The median (interquartile range) serum TNF- $\alpha$ level was 23 (3.9-67) $\mathrm{pg} \cdot \mathrm{mL}^{-1}$ in the study group and $2(1.6-5.97) \mathrm{pg} \cdot \mathrm{mL}^{-1}$ in the control group $(p=0.0001)$ (fig. 2$)$. The median serum IL-8 level was $34(7.2-49) \mathrm{pg} \cdot \mathrm{mL}^{-1}$ in the study group and $4(2.98$ 6.47) $\mathrm{pg} \cdot \mathrm{mL}^{-1}$ in the control group $(\mathrm{p}=0.0001)$ (fig. 3$)$. In repeatability tests, the interclass coefficient was 0.956 for TNF$\alpha$ (95\% CI: 0.870-0.985) and 0.925 for IL-8 (95\% CI: 0.779-0.975).

There was a significant positive correlation between TNF- $\alpha$ and IL-8 levels $(r=0.9, p<0.001)$ for both groups. In the study group, a significant negative correlation was observed between the serum level of IL- 8 and the impairment of lung function, as

\begin{tabular}{|c|c|c|c|}
\hline TABLE 3 & $\begin{array}{l}\text { ans of pulm } \\
\text { ly and cont }\end{array}$ & $\begin{array}{l}\text { ary function te } \\
\text { groups }\end{array}$ & 'ariables of \\
\hline Variable & Study group & Control group & $p$-value \\
\hline FVC \% pred & $93.1 \pm 9.9$ & $99.0 \pm 15.3$ & 0.02 \\
\hline FEV 1 \% pred & $92.9 \pm 11.8$ & $97.5 \pm 9.3$ & 0.04 \\
\hline FEV $_{1} /$ FVC $\%$ & $85.7 \pm 7.6$ & $87.8 \pm 6.1$ & $>0.05$ \\
\hline FEF25-75 \% pred & $77.6 \pm 16.4$ & $83.0 \pm 6.4$ & 0.04 \\
\hline DL,CO \% pred & $75.8 \pm 11.9$ & $85.6 \pm 9.9$ & $<0.001$ \\
\hline
\end{tabular}

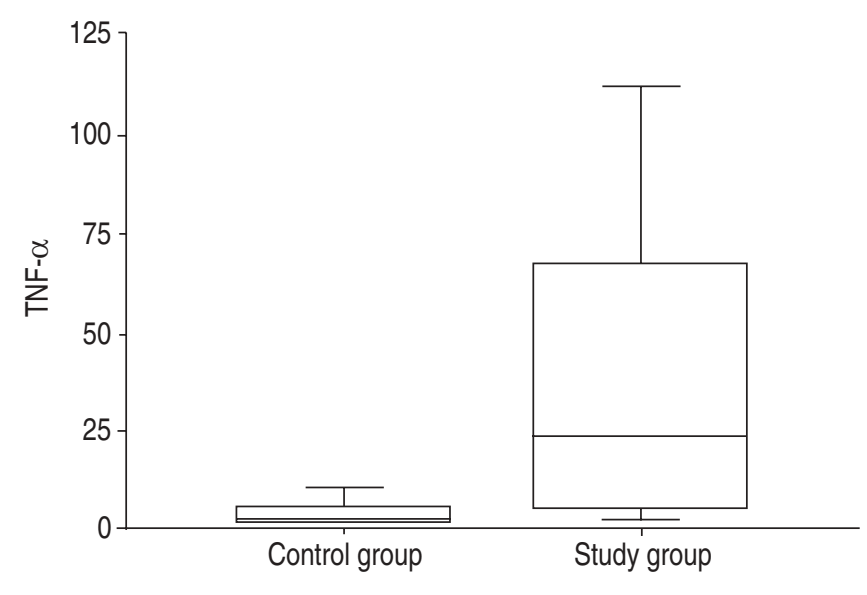

FIGURE 2. Serum tumour necrosis factor (TNF)- $\alpha$ levels of the two groups.

reflected by $D \mathrm{~L}, \mathrm{CO} \quad(\mathrm{r}=-0.44, \mathrm{p}=0.004)$. There was also a significant negative correlation between TNF- $\alpha$ levels and $D \mathrm{~L}, \mathrm{CO}(\mathrm{r}=-0.2, \mathrm{p}=0.01)$.

Chest radiographs of all subjects in the present study and control groups were evaluated as normal. Inspiratory and expiratory sections of HRCT, which was done in 10 polypropylene flocking workers with functional impairment (specify: low $\mathrm{DL}, \mathrm{CO})$, were interpreted as normal in one subject, showing a slight increase of bronchial shadows in two subjects, diffuse ground glass appearances in two subjects, air trapping areas in two subjects and thickened bronchial walls mainly in the lower lung fields in three workers (table 4, figs $4 \mathrm{a}$ and $4 \mathrm{~b})$.

Microscopic examination of polypropylene flock showed that the diameter of fibres was $6.9 \pm 2.1 \mu \mathrm{m}$ and the length of fibres was $96.7 \pm 35.2 \mu \mathrm{m}$. Scanning electron microscopy of the polypropylene-flock material showed irregular particles in shape and size as well as lamellar fragmentation of the surfaces (figs $5 a$ and $5 b$ ).

The inhalable and respirable dust concentrations in the flocking department were $4.4 \mathrm{mg} \cdot \mathrm{m}^{-3}$ and $<0.2 \mathrm{mg} \cdot \mathrm{m}^{-3}$, respectively. The $0.2 \mathrm{mg} \cdot \mathrm{m}^{-3}$ concentration value was just the

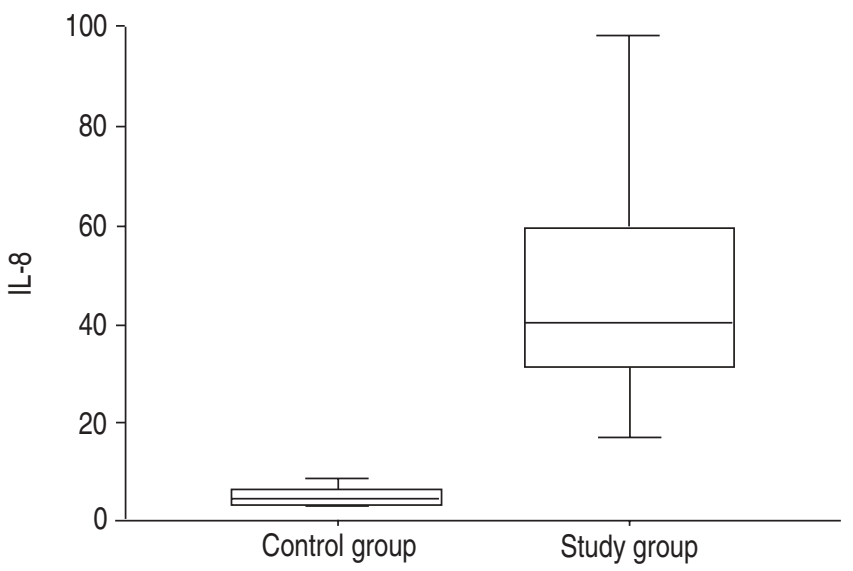

FIGURE 3. Serum interleukin (IL)-8 levels of the two groups. 


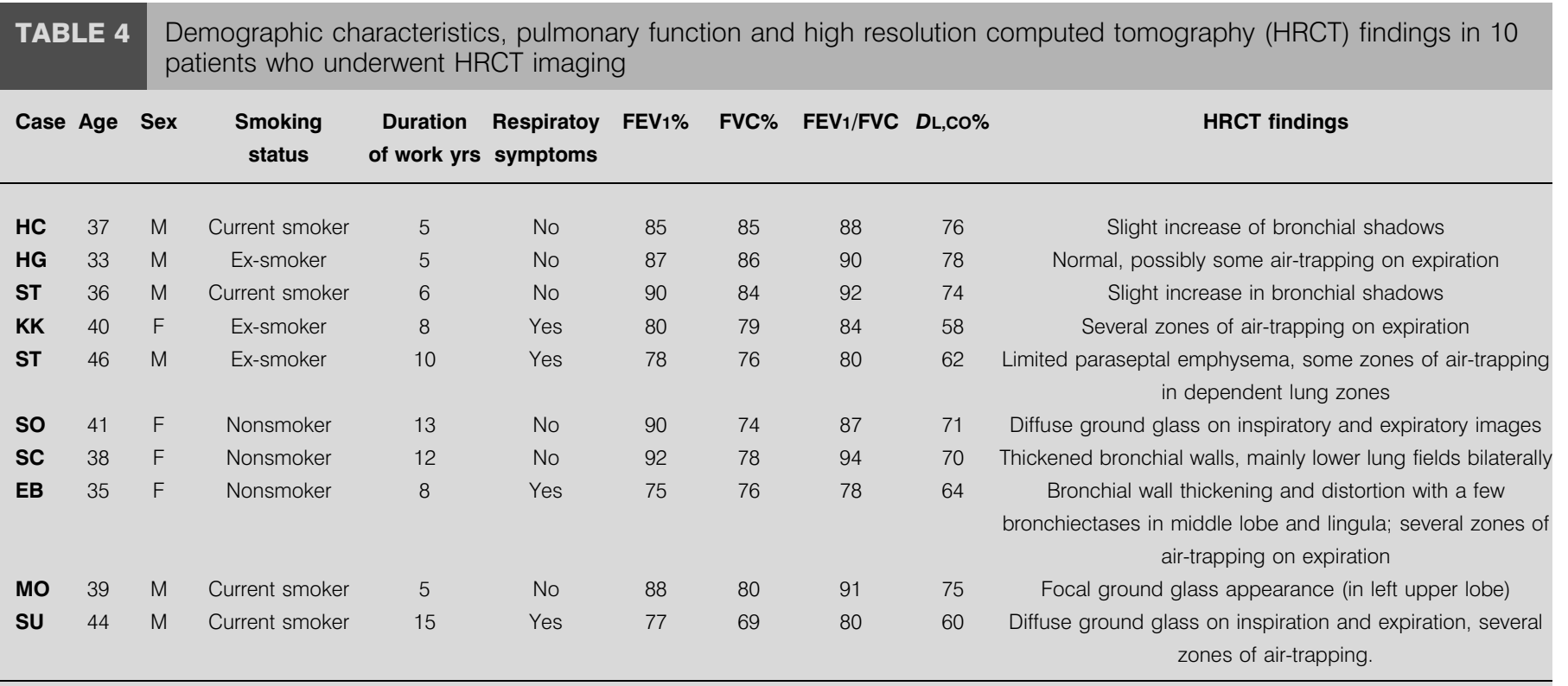

M: male; F: female; FEV1: forced expiratory volume in one second; FVC: forced vital capacity; $D L, C O$ : carbon monoxide diffusing capacity.

detectable limit of the sampling procedure, applied in relation to the sampling period and digital balance weighing resolution.

\section{DISCUSSION}

To the knowledge of the present authors, this is the first crosssectional study in which respiratory symptoms and pulmonary function have been evaluated in a large group of workers from a polypropylene flocking plant. Pulmonary function tests suggested the presence of restrictive impairment with decreased diffusion capacity in some workers exposed to polypropylene flock. These findings, together with HRCT in a limited number of subjects, are indicative of the beginning of or mild ILD, even though this was not accompanied by invalidating respiratory symptoms. Increased serum concentrations of IL- 8 and TNF- $\alpha$ were also found in these workers, and the levels of these cytokines were correlated with the decrease in diffusion capacity.

Several cases of overt occupational ILD, referred to as "flock worker's lung", have been reported among flock processing workers in recent years [1-4]. In general, these were symptomatic patients and further clinical investigation into them led to a diagnosis of ILD [1-4,6]. The first case of polyethylene flock-associated ILD was reported by BARROso et al. [4] in a woman who presented with a 12 months history of dyspnoea on effort and dry cough after a 7-year period of occupational exposure to rotary cut polyethylene [4]. In a cross-sectional study carried out by the US National Institute of Occupational Safety and Health, a high frequency of respiratory and systemic symptoms was reported in nylon flocking workers, with the symptom prevalence being related to the number of days worked per week [6]. In the present study, the prevalence of reported respiratory symptoms did not differ statistically between the polypropylene flocking workers and controls. However, logistic regression analysis showed that the risk of respiratory symptoms increased 3.6 fold in polypropylene flocking workers when compared to controls, whereas age, sex, tobacco use and working years were not found to be significantly associated with respiratory symptoms. In addition, polypropylene flocking workers also reported improvement when away from work at a higher rate than control workers (26\% versus $13 \%)$. The findings of the current authors are partly consistent with the epidemiological study of the National Institute for Occupational Safety and Health investigators [6].

In the only case of flock-associated ILD due to polyethylene exposure reported to date, pulmonary function testing revealed a severe restrictive functional defect [4]. In a study evaluating pulmonary function among nylon flocking workers, restriction was observed in $7.1 \%$ of production workers compared to $3.1 \%$ of office workers and low diffusing capacity was observed in $13.2 \%$ of production workers compared to $5.3 \%$ of the office workers [6]. Consistent with these observations, the present study found a significantly higher rate of (mild) restrictive impairment and reduced diffusion capacity in the polypropylene flocking workers when compared to controls. Restriction was found in $20 \%$ of polypropylene flocking workers compared to $4.4 \%$ of the controls and a low diffusing capacity was observed in $26 \%$ of polypropylene flocking workers compared to $4.4 \%$ of the control group. Additionally, MANOVA showed that working with the polypropylene flock and the duration of work were predictive factors for impairment of lung function, whereas tobacco use was not.

In most diffuse lung diseases, imaging and functional abnormalities are central to clinical evaluation [18]. None of the workers had significant abnormalities on chest radiographs, indicating that there was no subject with established ILD or fibrosis. The present authors could not justify performing HRCT in all subjects, so this was only done in 10 selected polypropylene flocking workers with functional 

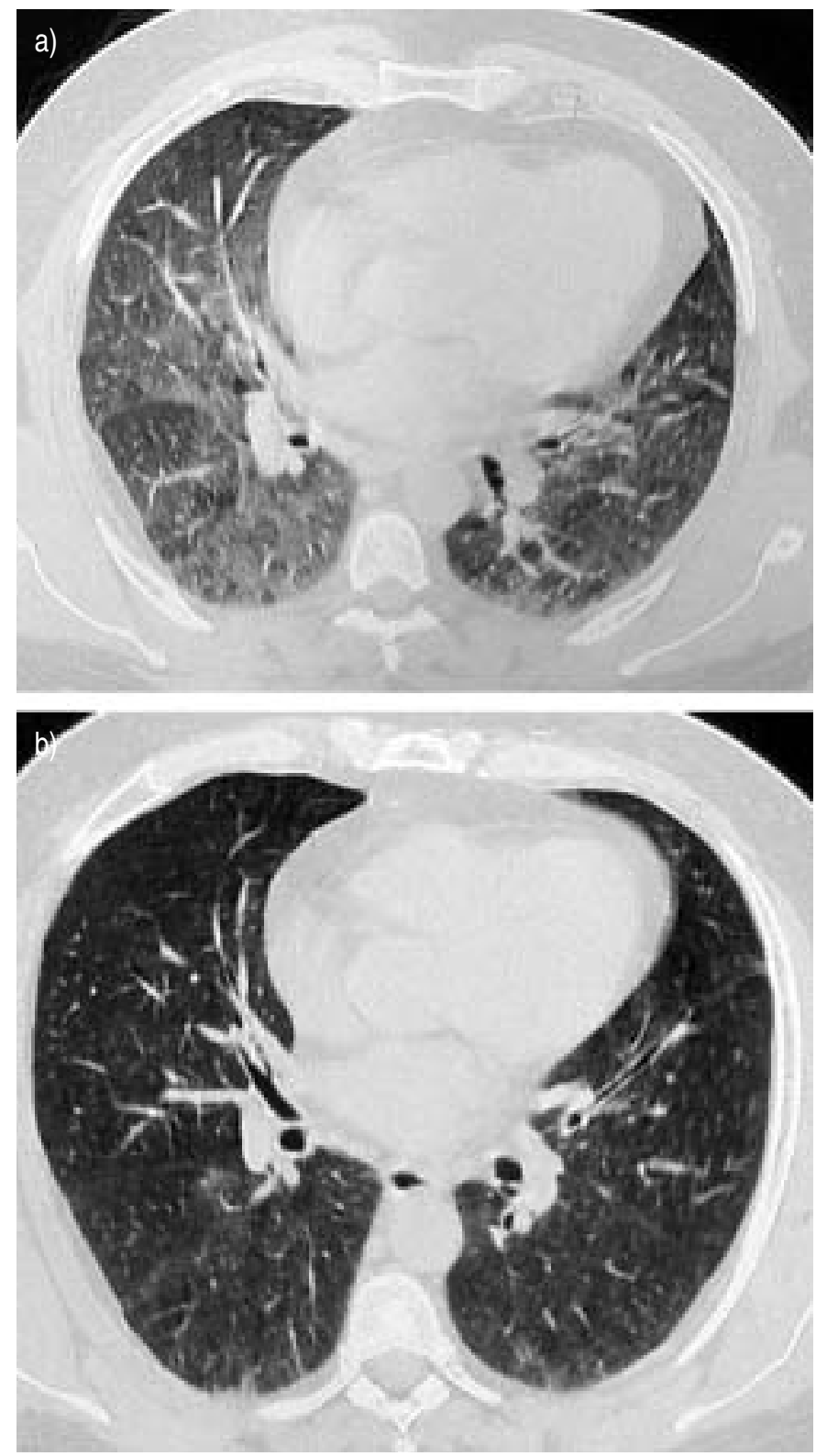

FIGURE 4. a) Inspiratory high-resolution computed tomography (HRCT) examination showing density increase, especially in the posterior subpleural region and peribronchial thickening. b) Expiratory HRCT examination showing air trapping areas indicating a distal airway disease.

impairment (i.e. reduced DL,CO). HRCT revealed mild, generally nonspecific abnormalities, some of which are compatible with ILD (diffuse ground glass opacities) and/or bronchiolitis (focal air trapping). The pathology of flock worker's lung has been characterised as lymphocytic bronchiolitis and peribronchiolitis with lymphoid hyperplasia, as concluded from a review of all lung biopsies [1-4, 6].

No previous study could be found which has assessed serum cytokines in the pathogenesis of flock worker's lung. It is well known that various cytokines secreted from macrophages play a key role in the pathogenesis of various pulmonary diseases. Increased IL-8 and TNF- $\alpha$ have been described in various inflammatory disorders characterised by neutrophilic
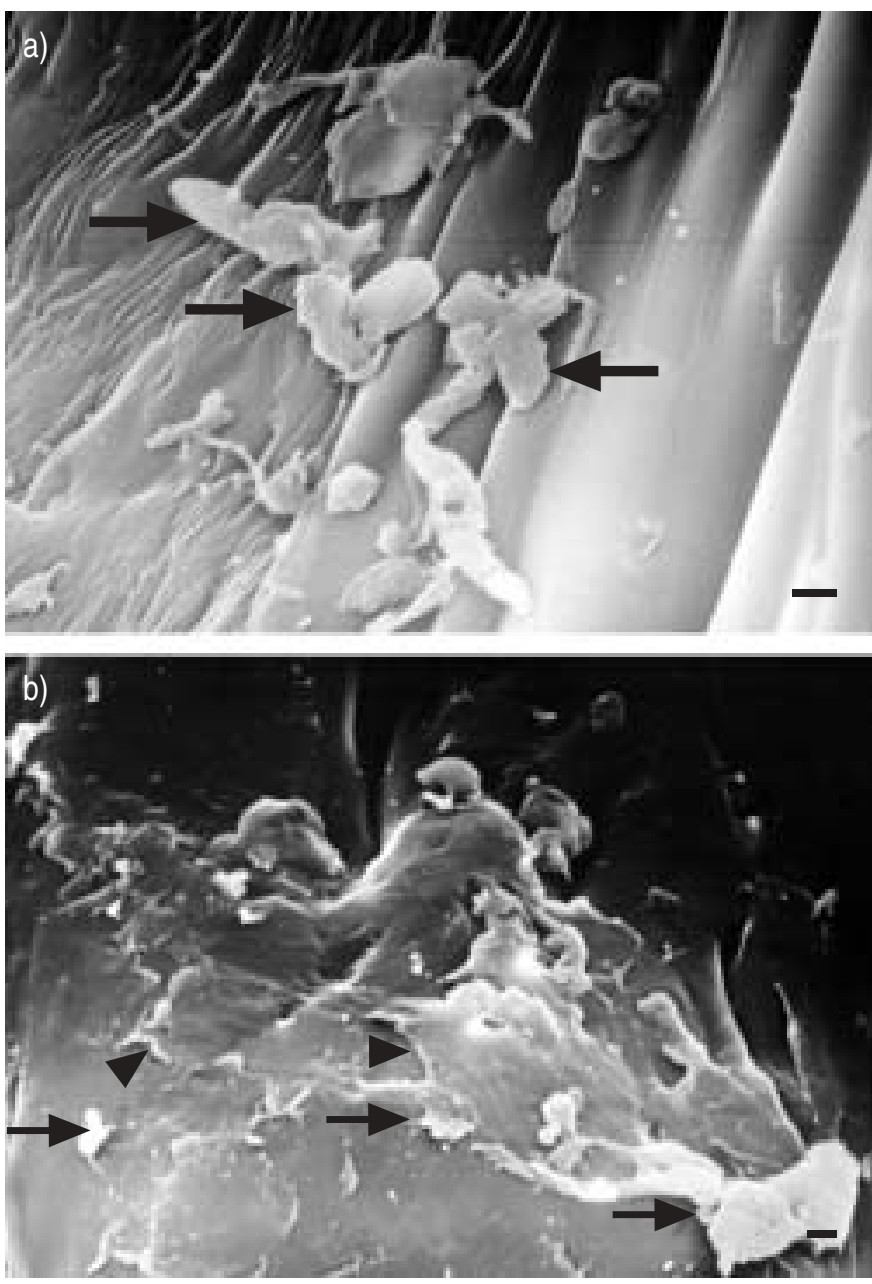

FIGURE 5. a) Scanning electron microscope (SEM) photomicrograph of the polypropylene-flock material showing irregular particles in shape and size scattered widely. Arrows show the particles. Scale bar $=10 \mu \mathrm{m}$. b) SEM photomicrograph of the polypropylene-flock material showing both the irregular particles (arrows) and the lamellar fragmentation of the surface (arrowheads) which seem fragile. Scale bar $=10 \mu \mathrm{m}$.

infiltration, including interstitial pulmonary disorders $[10,12$, 13]. Toxicological studies suggest that respirable fragments of nylon might induce an intense inflammatory lung injury characterised by increased polymorphonuclear neutrophils $[7,19]$. Thus far, only one study is available concerning the inhalation toxicity of polypropylene fibre. In that experimental study, microscopic examination of polypropylene fibreexposed lungs revealed a dose-dependent increase in cellularity (especially macrophage infiltration), but no fibrosis [5]. Here, the current authors found high serum IL- 8 and TNF- $\alpha$ levels in the polypropylene flocking workers. Furthermore, these levels showed a negative correlation with $D \mathrm{~L}, \mathrm{CO}$. These results suggest that IL- 8 and TNF- $\alpha$ may act as key mediators for inflammatory lung injury in the pathogenesis of polypropylene flocking worker's lung.

The exact aetiological agent for flock worker's lung is still unknown. To date, the results of NIOSH laboratory studies appear to incriminate respirable-sized nylon particulate as an aetiological agent for flock worker's lung [7, 8]. Recently, 
polypropylene flock has been described as causing flockassociated ILD [4]. In the present study, raw material of flock in the investigated plant was composed of $90 \%$ polypropylene and $5 \%$ polyethylene. Flock is produced by guillotine cutting or rotary cutting. Rotary cutting has been claimed to be more hazardous than guillotine cutting $[1,4]$. In the present study, polypropylene flock was produced by guillotine cutting in this particular plant.

During the flocking process, respirable-sized particles (aerodynamic diameter $<10 \mu \mathrm{m})$ may be released [8, 9]. The diameter of propylene fibre depends on the manufacturing process and can vary from $>153 \mu \mathrm{m}$ in monofilament yarn to an average of $1-5 \mu \mathrm{m}$ in microfibres [5]. In the present study, microscopic examination of polypropylene flock particles showed that the diameter of the fibres was $6.9 \pm 2.1 \mu \mathrm{m}$ and the length of the fibres was $96.7 \pm 35.2 \mu \mathrm{m}$. A wide variety of fibres and particles should now be accepted as being pathogenic when the susceptible individual is exposed to the "right" concentrations for the "right" duration [20]. It was reported that, within this industry, facilities having higher air concentrations of respirable dust are found to have correspondingly higher rates of ILD [1]. In an experimental study assessing pulmonary toxicity of inhaled polypropylene fibres in rats, HESTERBERG et al. [5] found that the severity of pulmonary injury appeared to increase with the dose and the duration of exposure. The mechanisms by which flock causes lung damage and possible fibrosis is not known. It is conceivable that following high exposures, the phenomenon described as "particle overload" or "dust overload" leads to impairment in particle clearance by alveolar macrophages resulting in a persistent excessive elaboration and release of mediators, leading to inflammation and fibrosis [21]. In this study, the ambient air concentrations of inhalable and respirable dust were found to be $<10 \mathrm{mg} \cdot \mathrm{m}^{-3}$ and $5 \mathrm{mg} \cdot \mathrm{m}^{-3}$, respectively, i.e. values often considered as limits for "nuisance" dusts [22]. Yet, clouds of polypropylene flock were clearly visible in the production halls, but this may be attributed to large particles $>100$ microns in size. Due to their aerodynamic characteristics, such large particles are not inhaled during normal breathing, although a fraction might be inhaled during occasional deep breaths. Additionally, it is believed that visible dust in the air suggests that the ventilation in the work area is inadequate [23]. Moreover, the sampling and gravimetric measurements of dusts made here may not have been totally adequate for assessing exposure to fibrous materials, such as flock. The subjects worked $8 \mathrm{~h}$ per day, and 6 days per week, without any respiratory protective measures. The current study underscores the importance of preventive measures in the work area. Exposure to flock associated dust should be reduced and controlled by adequate engineering measures, complemented by effective personal respiratory protection in high dust areas.

A limitation of the present study is the probable occurrence of a healthy worker effect, which is a common bias in crosssectional occupational studies, and is probably a source of underestimation of the effect of exposure [24]. This may explain partly why only subclinical pulmonary disease was found among the study group. No information was available about whether any worker ever left the factory because of respiratory disease or was seen elsewhere as a patient with
ILD. Another likely reason for the absence of overt clinical disease in the current study is the relatively young age of the studied population. The latter is probably also the reason for the lack of significant effect of smoking.

In conclusion, the present authors found exposure to polypropylene flock in the workplace to be associated with pulmonary functional impairment of a restrictive type and with reduced diffusing capacity. The increased serum interleukin- 8 and tumour necrosis factor- $\alpha$ levels in the polypropylene flocking workers are indicative of an ongoing pro-inflammatory process in these subjects and this may be relevant in the pathogenesis of flock worker's lung. Appropriate medical surveillance and exposure control should be provided in the polypropylene flock industry.

\section{ACKNOWLEDGEMENTS}

The authors would like to thank H. Çelik and İ. Tatar from Hacettepe University School of Medicine and H. Ozturk from Mersin University School of Medicine for electron microscopic examination, K. Tasli from Mersin University School of Engineering for light microscopic examination, J. Verschakelen from the U.Z. Gasthuisberg in Leuven for reviewing the HRCT scans, S. Ozcan for his help in carrying out the dust measurements at the plant, and A. Cimrin for his valuable suggestions about the study.

\section{REFERENCES}

1 Kern DG, Kuhn C 3rd, Ely EW, et al. Flock worker's lung: broadening the spectrum of clinicopathology, narrowing the spectrum of suspected etiologies. Chest 2000; 117: 251-259.

2 Eschenbacher WL, Kreiss K, Lougheed MD, Pransky GS, Day B, Castellan RM. Nylon flock-associated interstitial lung disease. Am J Respir Crit Care Med 1999; 159: 2003-2008.

3 Kern DG, Crausman RS, Durand KT, Nayer A, Kuhn C 3rd. Flock worker's lung: chronic interstitial lung disease in the nylon flocking industry. Ann Intern Med 1998; 129: 261-272.

4 Barroso E, Ibanez MD, Aranda FI, Romero S. Polyethylene flock-associated interstitial lung disease in a Spanish female. Eur Respir J 2002; 20: 1610-1612.

5 Hesterberg TW, McConnell EE, Miiller WC, Hamilton R, Bunn WB. Pulmonary toxicity of inhaled polypropylene fibers in rats. Fundam Appl Toxicol 1992; 19: 358-366.

6 Washko RM, Day B, Parker JE, Castellan RM, Kreiss K. Epidemiologic investigation of respiratory morbidity at a nylon flock plant. Am J Ind Med 2000; 38: 628-638.

7 Porter DW, Castranova V, Robinson VA, et al. Acute inflammatory reaction in rats after intratracheal instillation of material collected from a nylon flocking plant. J Toxicol Environ Health A 1999; 57: 25-45.

8 Burkhart J, Piacitelli C, Schwegler-Berry D, Jones W. Enviromental Study of Nylon Flocking Process. J Toxicol Environ Health A 1999; 57: 1-23.

9 Burkhart J, Jones W, Porter DW, Washko RM, Eschenbacher WL, Castellan RM. Hazardous occupational exposure and lung disease among nylon flock workers. Am J Ind Med, 1999, Suppl. 1, 145-146.

10 Ziegenhagen MW, Zabel P, Zissel G, Schlaak M, MullerQuernheim J. Serum level of interleukin 8 is elevated 
in idiopathic pulmonary fibrosis and indicates disease activity. Am J Respir Crit Care Med 1998; 157: 762-768.

11 Vaillant $\mathrm{P}$, Menard O, Vignaud JM, Martinet N, Martinet Y. The role of cytokines in human lung fibrosis. Monaldi Arch Chest Dis 1996; 51: 145-152.

12 Lynch JP, Standiford TJ, Rolfe MW, Kunkel SL, Strieter RM. Neutrophilic alveolitis in idiopathic pulmonary fibrosis. The role of interleukin-8. Am Rev Respir Dis 1992; 145: 1433-1439.

13 Kunkel SL, Standiford T, Kasahara K, Strieter RM. Interleukin-8 (IL-8): the major neutrophil chemotactic factor in the lung. Exp Lung Res 1991; 17: 17-23.

14 Burney PGJ, Chinn S, Britton JR, Tattersfield AE, Papacosta AO. What symptoms predict the bronchial response to histamine? Evaluation in a community survey of the bronchial symptoms questionnaire (1984) of the international union against tuberculosis and lung diseases (IUATLD). Int J Epidemiol 1989; 18: 165-173.

15 Quanjer PH, Tammeling GJ, Cotes JE, Pedersen OF, Peslin R, Yernault JC. Lung volumes and forced ventilatory flows. Report of the Working Party on Standardisation of Lung Function Tests, European Community for Steel and Coal. Official Statement of the European Respiratory Society. Eur Respir J 1993; 6: Suppl. 16, 5-40.

16 Cotes JE, Chinn DJ, Quanjer PH, Roca J, Yernault JC. Standardisation of the measurement of transfer factor
(Diffusing capacity). Report of the Working Party on Standardisation of Lung Function Tests, European Community for Steel and Coal. Official Statement of the European Respiratory Society. Eur Respir J 1993; 6: Suppl. $16,41-52$.

17 Broaddus VC, Hebert CA, Vitangcol RV, Hoeffel JM, Bernstein MS, Boylan AM. Interleukin-8 is a major neutrophil chemotactic factor in pleural liquid of patients with empyema. Am Rev Respir Dis 1992; 146: 825-830.

18 Hansell DM. Computed tomography of diffuse lung disease: functional correlates. Eur Radiol 2001; 11: 1666-1680.

19 Merrill WW. Flock worker's lung. Ann Intern Med 1999; 130: 615-616.

20 Kuschner WG. What exactly is flock worker's lung? Chest 2000; 117: 10-13.

21 Morrow PE. Dust overloading of the lungs: update and appraisal. Toxicol Appl Pharmacol. 1992; 113: 1-12.

22 McLaughlin JK, Lipworth L, Marano DE, Tarone R. A critical evaluation of the scientific basis of the MAK Commission's new general threshold limit values for dust. Int Arch Occup Environ Health 2001; 74: 303-314.

23 Balmes JR. Occupational respiratory diseases. Prim Care 2000; 27: 1009-1038.

$24 \mathrm{Li} \mathrm{CY}$, Sung FC. A review of the healthy worker effect in occupational epidemiology. Occup Med 1999; 49: 225-229. 